\title{
DETERMINANTES DEL USO DEL CRÉDITO DE VIVIENDA POR PARTE DE LOS HOGARES BOGOTANOS
}

Determinants of the use of mortgage credit by households in Bogota

Milton Samuel Camelo Rincón, Jonny Steven Amaya Cárdenas, John Fredy Parra Guzmán 


\section{DETERMINANTES DEL USO DEL CRÉDITO DE VIVIENDA POR PARTE DE LOS HOGARES BOGOTANOS}

\section{Determinants of the use of mortgage credit by households in Bogota}

Milton Samuel Camelo Rincón ${ }^{\mathrm{a}}$, Jonny Steven Amaya Cárdenas ${ }^{\mathrm{b}}$, John Fredy Parra Guzmán ${ }^{\mathrm{c}}$

Keywords: Mortgage Credit, Financing, Logit Model

Palabras clave: Crédito hipotecario, Financiamiento, Modelo Logit

JEL Classification: D14, H31, G21

Received: 20/06/2018

Accepted: 04/05/2019

Published: 26/06/2019 a. Universidad de la Salle, Bogotá Colombia mscamelo@unisalle.edu.co Orcid: 0000-0001-8727-1257

b. Universidad de la Salle, Bogotá Colombia jamaya52@unisalle.edu.co Orcid: 0000-0003-1504-3241

c. Universidad de la Salle, Bogotá Colombia jparra47@unisalle.edu.co

Orcid: 0000-0001-9789-3025

\section{Resumen}

El crédito hipotecario es reconocido en Colombia como una de las herramientas más utilizadas para obtener vivienda propia. Esta investigación identifica los principales determinantes del crédito hipotecario, y estima la influencia de factores como la educación, el ingreso y el empleo en la probabilidad de hacer uso efectivo de tal crédito por parte de los hogares bogotanos. Con tal propósito, se realiza una descripción del marco normativo del acceso a la vivienda y las condiciones de financiación establecidas por las principales entidades bancarias. Posteriormente, y con base en los datos recolectados por la encuesta multipropósito, realizada en 2014, para Bogotá, se estima un modelo Logit para establecer la probabilidad de uso del crédito para la compra de vivienda. Los resultados sugieren que la edad, el ingreso y la estabilidad laboral influyen significativamente sobre la decisión de uso de crédito; sin embargo, el factor más importante está dado por el nivel de educación.

\section{Abstract}

Mortgage credit is recognized in Colombia as one of the most used tools to obtain homeownership. This research identifies the main determinants of mortgage credit and estimates the influence of factors such as education, income and employment in the probability of making effective use of such credit by households in Bogota. In order to aim that purpose, this paper makes a description of regulatory framework for access to housing and the financial conditions established by major banks. Subsequently, and bases on the data gathered by the multipurpose survey made in 2014 for Bogota, a Logit model 
was estimated to establish the probability of use of credit for the purchase of housing. The results suggest that age, income and job stability influence the decision to use mortgage credit, however, the most important factor is given by the level of education.

El acceso a la vivienda propia constituye uno de los mayores desafíos que debe enfrentar un hogar en Colombia. La Constitución Política del país establece el derecho de todo colombiano a acceder a una vivienda, y la Ley 546 de 1999 busca estimular su demanda bajo mecanismos de ahorro, subsidios y financiación; sin embargo, estas herramientas parecen ser insuficientes. Según datos del censo general realizado en Colombia en el año 2005, el número de hogares en déficit de vivienda era de 3828 o55, significando el $36 \%$ del total. Además, en las últimas décadas, el crecimiento poblacional y la escasez de la tierra han generado una serie de restricciones desde la oferta que han llevado a crecimientos en los precios de la vivienda superiores al índice general de precios (IPC).

A pesar de existir cierto consenso en que se trata de un problema estructural desde la oferta (Camelo 83 Ocampo, 2016), el crédito hipotecario se ha convertido en el mecanismo habitual por el cual los hogares optan para financiar su compra de vivienda, el cual es ofrecido por entidades como el Fondo Nacional del Ahorro (FNA) y la banca comercial (entidades autorizadas por el Gobierno Nacional para ofrecer este tipo de financiación). Si bien la estrategia de financiamiento presenta virtudes, el crédito hipotecario cuenta con cierto número de características y requisitos que instigan a las entidades oferentes a racionarlo, restringiendo la posibilidad de las familias de ser propietarias de vivienda. Adicionalmente, como ya se mencionó, los estímulos a la demanda están lejos de resolver los problemas del mercado de vivienda, cuyas características son muy diferentes a las de un bien privado tradicional.

Finalmente, a pesar de la existencia de algunos estudios sobre financiamiento de vivienda, hay ausencia de investigaciones realizadas sobre el acceso a la figura de crédito hipotecario en la ciudad de Bogotá, donde la heterogeneidad de su población y la amplia gama de alternativas financieras permiten obtener evidencias sobre los factores socioeconómicos más importantes. Es así como la presente investigación tuvo como propósito identificar, de manera más precisa, la propensión a utilizar el crédito hipotecario en esta ciudad, según las características socioeconómicas del jefe de hogar. Los resultados son evaluados a la luz de la teoría económica y derivan algunas recomendaciones de política.

Con el anterior propósito, este documento se organiza en cinco secciones, además de la presente introducción: en la primera, se discuten algunos antecedentes de literatura especializada sobre el tema; en la segunda, se realiza una breve revisión de postulados teóricos que pueden relacionarse con la problemática; en la tercera, se realiza una descripción del marco normativo del acceso a la vivienda y las condiciones de financiación establecidas por las principales entidades bancarias; en la cuarta, se estima un modelo Logit para establecer la propensión al uso del crédito para la compra de vivienda en función de las diferentes variables consideradas; y, en la última sección, se plantean algunas conclusiones y recomendaciones. 


\section{Antecedentes de literatura}

El tema de vivienda es de permanente relevancia en los análisis académicos, pero, sobre todo, en los de política pública. La discusión es de vieja data, alcanzando publicaciones clásicas tales como Friedman (1957), De Leeuw (1971), Poterba (1984), entre otros. El factor común de estos primeros aportes son las imposiciones que implican, para los hogares, las limitaciones presupuestales, es decir, el ingreso.

Aportes posteriores, como el de Fallis y Smith (1984), ponen en relieve algunas particularidades de este mercado, el alto control que implica y el acceso a los recursos vía préstamos bancarios. Esta dinámica, como plantea Connolly (1997), ha complejizado las relaciones entre prestamistas y deudores, quienes no tienen claros incentivos al ofrecer los recursos para este fin, ni al pagar sus deudas, respectivamente. En esta línea de argumentación también están Daphnis (2006) y Young (2007), quienes, en cierta forma, sugieren que las entidades financieras no suelen estar interesadas en disponer recursos para el financiamiento de la compra de vivienda, pues la población objeto de estos créditos no tiende a brindar garantías sobre su retorno.

En cuanto a investigaciones más recientes, la producción científica al respecto ha decaído en los últimos años, siendo el principal enfoque de discusión el aspecto financiero. Así, como se observa en Ferguson y Navarrete (2003), se puede decir que hay cierto consenso sobre la importancia de la vivienda en la calidad de vida de las familias; sin embargo, no se ha avanzado significativamente en propuestas efectivas para su regulación y financiación, pues existen tensiones entre el derecho a la vivienda digna y la autonomía de las entidades financieras privadas (Baena, 2017).

En efecto, en la literatura se encuentra una importante cantidad de trabajos sobre vivienda y, para el caso de Bogotá, en algunos de estos se entiende que la posesión de vivienda determina el bienestar del individuo e, incluso, la no posesión de la misma se asocia con el concepto de pobreza. Para el caso específico de Bogotá, Camargo y Hurtado (2011) destacan factores como la informalidad y los bajos salarios como determinantes del bajo acceso a vivienda propia; sin embargo, mencionan que en los últimos años se ha frenado la tendencia hacia la vivienda informal y la expansión hacia las periferias de la ciudad. Torres, Rincón y Vargas (2009), en un estudio profundo de la pobreza urbana en la ciudad, abordan la problemática de forma semejante, entendiendo que el mejoramiento integral de la vivienda es necesario para superar el déficit cualitativo de la ciudad de Bogotá. Este aspecto tiene que ver con la necesidad que los gobiernos locales han enfrentado por generar planes de ordenamiento territorial.

Otros trabajos se enfocan en los determinantes del financiamiento, el cual, siguiendo a Gonzales (2005), depende de dos características: la rentabilidad para el prestatario y la adecuada capacidad de pago por parte del deudor. Díaz (2011) reconoce al sistema bancario como el principal proveedor de préstamos a los hogares en economías poco desarrolladas; y es en tal punto donde nace la figura del racionamiento de crédito. Dicho racionamiento se da debido a las múltiples condiciones que imponen las entidades financieras para garantizar el retorno de sus recursos, más una utilidad en forma de intereses. Para Villareal (2010), en torno a estas condiciones, existe un "engaño ex ante" generado por la información asimétrica propia de los mercados financieros.

Clavijo, Muñoz y Janna (2004) han realizado modelaciones del crédito de vivienda, donde observan que los factores como ingreso de los hogares y precio de las viviendas afectan las decisiones a la hora de tomar un crédito hipotecario, observando altas elasticidades en sus estimaciones. Por su parte, Murcia (2007) critica el hecho de que, en Colombia, la mayoría de trabajos abordan este tema desde la perspectiva macroeconómica, por lo que destaca que el enfoque microeconómico permite identificar 
factores como el ingreso, la posición geográfica, el nivel de educación, el acceso a la seguridad social y la edad. Este argumento coincide con Hood (1999), quien considera tan importante la variable educación como la presencia de niños en el hogar, pues hay un efecto sustitución entre vivienda y educación de los niños.

Metodológicamente, cuando se consideran variables microeconómicas, los modelos probabilísticos suelen ser utilizados para evaluar los determinantes del acceso a vivienda de un hogar. Carvajal, García y Cotte (2015) encontraron, mediante un modelo Probit, que los principales determinantes de acceso a vivienda eran el ingreso del hogar, el ahorro programado, el número de miembros del hogar, la edad del jefe de hogar, el valor del arriendo que se pagaba y los años de vivir en arriendo. De otro lado, según Rojas, Bran y Rincón (2013), estas variables pueden diferir dependiendo de la región y el país.

Esta mirada a la literatura requiere destacar algunos trabajos a nivel regional, pues parece evidente que la problemática se extiende a la mayoría de países latinoamericanos. Serageldin y Driscoll (2000) realizaron una serie de estudios de caso en Asia, África y Latinoamérica, encontrando la importancia de los microcréditos como iniciativas para suplir las necesidades de los hogares de escasos recursos. Ferguson y Smets (2010) también destacan este tipo de iniciativas, sugiriendo que la tendencia es a expandir el destino de los recursos hacia el mejoramiento y formalización de las viviendas existentes. Estos esfuerzos son evidentes si se consideran las múltiples propuestas que, desde la academia, el sector público y las organizaciones no gubernamentales, se han desarrollado en cada país para promover el acceso a la vivienda digna (Fruet 83 Muñoz, 2015; Rodríguez 83 Sugranyes, 2012; Serrano, 2012; Durán, 2013).

Finalmente, mientras la tendencia hacia el predominio del crédito bancario (hipotecario) como fuente de financiación de vivienda no se revierta -lo cual aún parece ser lejano-, es fundamental establecer en qué medida los hogares son más propensos a hacer uso de él y cuáles son los determinantes de tal uso. El análisis empírico que se desarrolló en esta investigación da algunas luces sobre ello.

\section{Alguna teoría relevante}

Esta investigación presenta un enfoque microeconómico, en el sentido que evalúa el comportamiento de los hogares según características del jefe de hogar y las condiciones establecidas por el sistema financiero. De ahí que es posible partir de la teoría del consumidor como referente y, posteriormente, enriquecer el análisis mediante el contraste de las asimetrías de información y la teoría del ciclo vital.

\section{Teoría del consumidor}

Esta teoría analiza el comportamiento de los agentes económicos (consumidores) que interactúan en determinados mercados y cuyo problema es alcanzar los niveles máximos de utilidad, sujetos a su restricción presupuestal. Dadas las características del mercado, los consumidores evalúan sus gustos y sus preferencias, y toman decisiones fundamentadas en la racionalidad, de tal manera que son capaces de comparar cestas de consumo adquiriendo aquellas que sean factibles y otorguen una mayor utilidad. Para los marginalistas, esta conducta implica que la utilidad que genera una unidad adicional de un bien debe equipararse con el costo de adquirirlo.

Generalmente, adquirir una vivienda significa enfrentarse a un bien de precio superior con relación a los ingresos potenciales de los hogares, por lo que contraen algún tipo de obligación (crédito hipotecario) para poder acceder a ella. Pero no todos los individuos acceden libremente al mercado de 
crédito. Gonzales (2005) afirma la existencia de tres obstáculos frente al acceso y financiamiento de vivienda: 1) su insuficiente capacidad adquisitiva no les permite cambiar su demanda potencial por una demanda efectiva; 2) los hogares carecen de garantías aplicables para obtener la financiación necesaria; y 3) en su mayoría, los países con sectores informales presentan la imposibilidad de acreditar ingresos formales y permanentes.

\section{Asimetrías de información}

Generalmente, los mercados de crédito influyen en y son influenciados por el resto de la economía. Gurley y Shaw (1955) resaltan la función de los intermediarios financieros a través de la oferta de crédito en la actividad económica, lo que consideran importante para la eficiencia de los mercados; sin embargo, a diferencia de los mercados competitivos donde no hay diferenciación y todos los agentes participan en igualdad de condiciones, la realidad demuestra que los sistemas de mercado son imperfectos o su comportamiento es diferente dada la normatividad, los derechos de propiedad, los costos financieros y de oportunidad, disponibilidad de la información, el volumen de los recursos y el alto número de agentes interactuando en él, entre otros, induciendo a los agentes participantes a tomar decisiones ineficientes, aumentando su incertidumbre.

Asimismo, estas estructuras de mercado traen consigo problemas de asimetrías de información que afectan de manera significativa la interpretación de datos por los agentes del mercado. Este problema implica riesgos tanto para prestamistas como para prestatarios. En efecto, $\underline{\text { Hoff y Stiglitz }}$ (1981) asumen que la información asimétrica influye en la racionalidad de los prestamistas, implicando riesgos, a tal punto que racionan los recursos disponibles entre los prestatarios solicitantes. Este es uno de los motivos más importantes que limitan el financiamiento, ya que, en muchas ocasiones, se oculta información importante para lograr determinar si quien requiere del crédito posee los medios para pagar la obligación con los intereses respectivos. El problema surge cuando uno de los agentes que se encuentra llevando a cabo la transacción posee información relevante desconocida por la otra, de forma que la primera realiza el proceso decisional y la transacción en superioridad de condiciones.

Akerlof (1970) denomina a este problema como una consecuencia de la selección adversa, al presentarse aquellas situaciones de "oportunismo precontractual" en las que, al iniciar o mantener un contrato, una de las partes (la que posee poca información) no tiene la capacidad de distinguir con toda seguridad la calidad de lo ofrecido por la otra parte. En este sentido, Stiglitz y Weiss (1981) establecen que dichos problemas de información afectan negativamente la eficiencia de los mercados de crédito, originando problemas de selección adversa, expresados desde las asimetrías de información y riesgo moral que conducen a los intermediarios financieros al racionamiento de crédito.

Dentro de la teoría neokeynesiana, se plantea que el riesgo moral es la causa ex post que deben enfrentar los intermediarios financieros. Vickrey (1961) hace referencia a los comportamientos "morales" de los agentes; en su obra utiliza el caso de una empresa donde los empleados (agentes) utilizan información privada privilegiada hacia el empresario contratante (principal), induciendo a generar un sistema de conductas veraces y comprometidas con el contrato de trabajo. En ese sentido, los oferentes se enfrentan a problemas de selección adversa para identificar y medir los niveles de riesgo para cada cliente, generando una tendencia al equilibrio del mercado; esto lleva a que la demanda de crédito no sea cubierta, estimulando la oferta con créditos de tasas bajas, atrayendo a los prestatarios de menor riesgo. 
La teoría del ciclo vital

Franco Modigliani (1986) es uno de los primeros teóricos que hizo referencia a la teoría de ciclo vital o función del consumo. En su investigación, considera que los individuos otorgan un ciclo natural a sus ingresos. Su planteamiento principal reside en que el consumidor representativo decidirá realizar un consumo razonable y estable, por lo que debe ser lo más cercano a la cantidad media que prevé consumir a lo largo de toda su vida. En ese sentido, el ingreso que perciben las personas tiende a variar sistemáticamente, de forma que, a lo largo de su vida, su comportamiento con respecto al ahorro y el consumo se encuentra determinado por su edad. Ando y Modigliani (1963) afirman que los ingresos percibidos por el trabajo poseen un perfil, y que a lo largo del tiempo es predecible, dadas las retribuciones máximas que se perciben en la edad adulta. Por ejemplo, cuando se nace o se pasa por la etapa de la niñez no se perciben altos ingresos; en la madurez, se perciben los ingresos necesarios para cumplir con la satisfacción de necesidades básicas (como puede ser la adquisición de vivienda y el ahorro); ya posteriormente, con la jubilación, el ingreso puede aumentar o disminuir, dependiendo del ahorro o ingreso generado para la posteridad. En efecto, la teoría del ciclo vital implica que las personas planifican su consumo y ahorro de acuerdo a grandes períodos de tiempo (juventud, adultez).

Por su parte, Friedman (1957) considera que el individuo tiende a suavizar su consumo prefiriendo mantenerlo estable, bajo un perfil uniforme de su renta a lo largo de su vida. Bajo este supuesto, se espera siempre recibir un ingreso permanente sin importar el momento del ciclo vital donde se encuentre; esta teoría es muy controvertida porque a medida que se va envejeciendo el poder adquisitivo disminuye y, al relacionarlo con esta investigación, la edad propicia para adquirir un crédito hipotecario es en las etapas juventud y adultez.

Modigliani (1986) concluye que los jóvenes son los que más dependerán del crédito; los adultos, los que más ahorrarán, y los ancianos, los que gastarán los ahorros que hicieron cuando fueron adultos. Pero, en la actualidad, esta teoría puede pasar a un segundo plano debido a que el ingreso, en muchos casos, no es el principal determinante para adquirir un crédito hipotecario, ya que en la juventud y a medida que se hace más adulto puede ser más riesgoso para una entidad financiera brindar un crédito hipotecario.

\section{Marco normativo del acceso a la vivienda y las condiciones de financiación}

El derecho a la vivienda digna se incorpora en el Artículo 51 de la Constitución de Colombia, de la siguiente manera:

Todos los colombianos tienen derecho a vivienda digna. El Estado fijará las condiciones necesarias para hacer efectivo este derecho y promoverá planes de vivienda de interés social, sistemas adecuados de financiación a largo plazo y formas asociativas de ejecución de estos programas de vivienda (Asamblea Nacional Constituyente, 1991).

De esta manera, se ha establecido un marco jurídico en el país que propende por el ejercicio y la responsabilidad del Estado como garante del acceso a la vivienda de sus ciudadanos. Sin embargo, dicho mandato posee una limitante, al ser un derecho de carácter asistencial, por lo que el Estado no está en la obligación de proporcionar unidades habitacionales a todos sus ciudadanos, relegando la función pública a la formulación de políticas de carácter financiero. En ese sentido, el crédito hipotecario en Colombia se rige por la Ley 546 de 1999, en la que se dictaron las normas más importantes en 
materia de financiación de vivienda para créditos de largo plazo y, en general, esta ley confiere las herramientas necesarias al Gobierno Nacional, para la regulación del mercado.

Los créditos hipotecarios son ofrecidos bajo dos modalidades o sistemas de amortización: pesos y UVR'. Estos sistemas fueron aprobados bajo la Ley 546 de 1999 y la Circular Externa o68 de 2000 (Superintendencia Financiera de Colombia [SFC], 2000a), en las que se estableció su instauración y, finalmente, su puesta en marcha por las diferentes entidades oferentes de crédito hipotecario. De la misma manera, y por disposición de la Circular Externa 085 de 2000 (SFC, 200ob), se autorizaron otras características aplicables a todo crédito con sistemas de amortización en UVR y pesos, como se muestra en la Figura 1.

Figura 1. Características de los sistemas de amortización en UVR y Pesos. Fuente: Elaboración propia
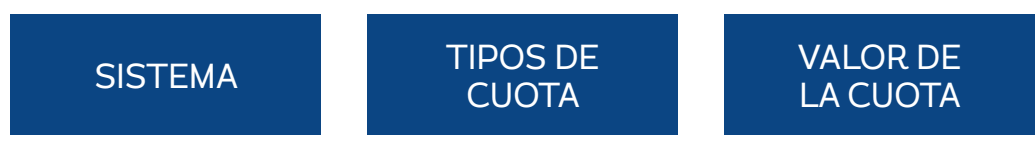

CARACTERÍSTICA

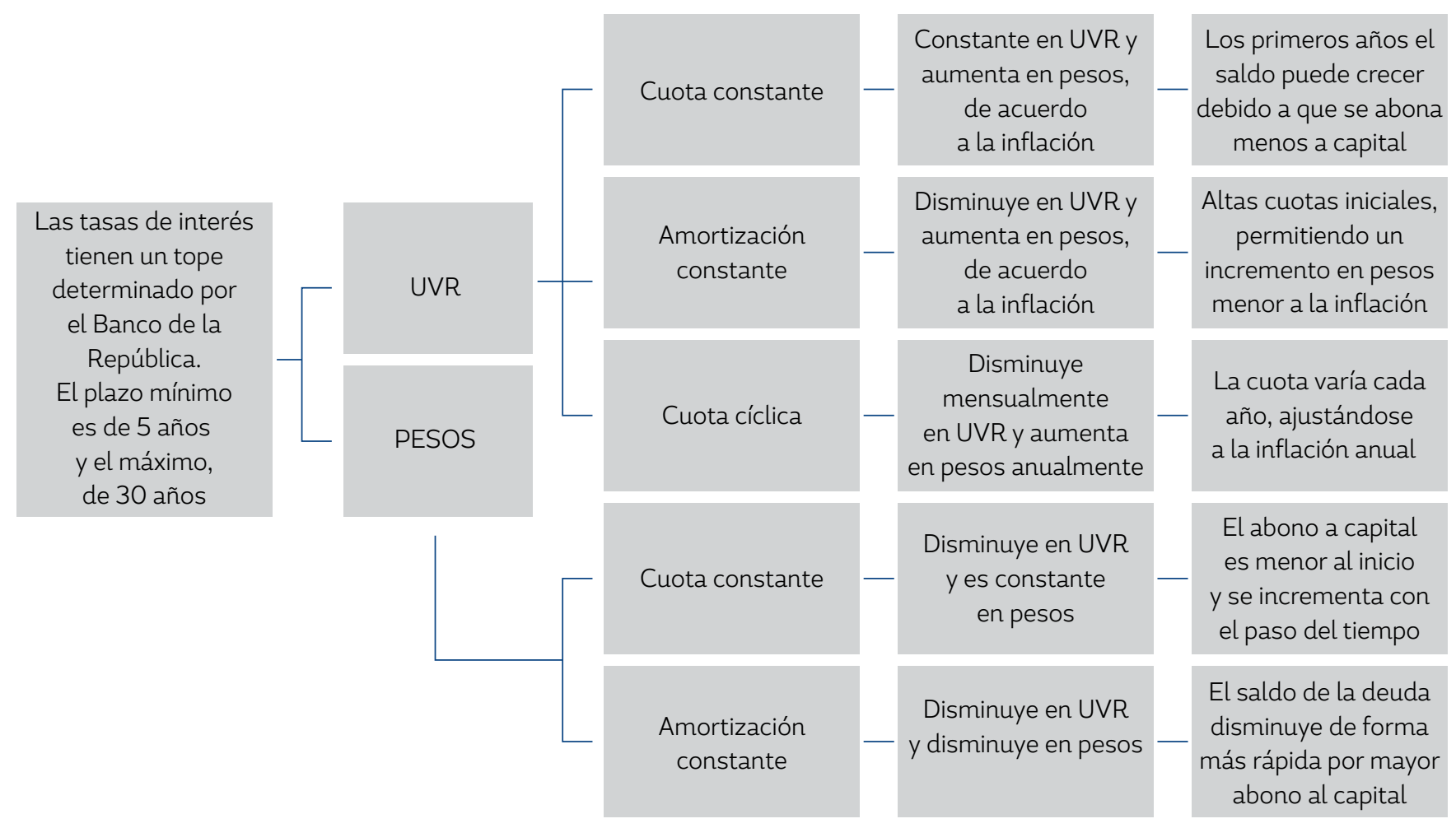

Además de las características mencionadas, se destacan las siguientes disposiciones respecto a los créditos hipotecarios:

- Tener un monto máximo sin exceder el porcentaje establecido por el Gobierno Nacional sobre el valor de la vivienda a financiar. Hoy en día, los montos solicitados como recursos iniciales oscilan entre $20 \%$ y $30 \%$ para vivienda VIS y no VIS.

- El crédito debe ser garantizado con hipoteca en primer grado del bien inmueble.

1 Un sistema de amortización es la forma como se estipula realizar el pago del préstamo, en cuotas mensuales que incluyen capital, intereses y seguros, y que se cancelan durante el plazo pactado. 
- La primera cuota del crédito no puede representar un porcentaje mayor a los ingresos familiares establecidos por el Gobierno Nacional. El valor fijado no puede superar el $30 \%$ de los ingresos demostrados (Decreto 145 de 2000).

- Los establecimientos de crédito están en la obligación de obtener y analizar la información de los deudores y la respectiva garantía. Además de ello, deben garantizar una metodología eficaz que permita evaluar el precio del inmueble a financiar, los ingresos del deudor, y que el crédito sea puntualmente atendido.

- Seguro de vida para el tomador del crédito que respalde la deuda ante la entidad en caso de deceso o incapacidad total. Además, seguro de incendio o terremoto para la protección del inmueble en caso de siniestro.

Las Tablas 1 y 2 ofrecen luces sobre las principales características y requisitos del crédito hipotecario, tomando como referencia las cinco entidades bancarias que presentaron el mayor índice de desembolsos en el año 2016.

Tabla 1. Características de los créditos hipotecarios en el sector financiero

\begin{tabular}{l|c|c|c|c|c}
\multirow{2}{*}{ Banco } & \multicolumn{2}{|c|}{ Plazos en años } & Porcentaje de financiación & Monto mínimo \\
\cline { 2 - 5 } & UVR & Pesos & VIS & No VIS & $70 \%$ \\
\hline Bancolombia & 5 a 30 & 5 a 15 & $80 \%$ & $70 \%$ & 20 \\
\hline Banco de Bogotá & 5 a 20 & 5 a 20 & $80 \%$ & $70 \%$ & 5 \\
\hline Banco Davivienda & 5 a 30 & 5 a 20 & $70 \%$ & $70 \%$ & 6 \\
\hline Banco BBVA & 5 a 20 & 5 a 20 & $70 \%$ & $70 \%$ & 20 \\
\hline Banco Caja Social & 5 a 20 & 5 a 15 & $70 \%$ & & 20 \\
\hline
\end{tabular}

Nota: elaboración propia a partir de las páginas web de las entidades bancarias.

Tabla 2. Requisitos para el estudio de crédito para empleados e independientes

\begin{tabular}{l|c|c|c|c}
\multirow{2}{*}{\multicolumn{2}{c|}{ Banco }} & \multicolumn{2}{c|}{ Rango de edad } & \multicolumn{2}{c}{ Ingresos mínimos (SMMLV) } \\
\cline { 2 - 5 } & Empleados & Independientes & Empleados & Independientes \\
\hline Bancolombia & 18 a 69 & 23 a 69 & 1,5 & 1,5 \\
\hline Banco de Bogotá & 18 a 70 & 18 a 70 & 1,5 & 1,5 \\
\hline Davivienda & 18 a 69 & 18 a 69 & 1,5 & 1,5 \\
\hline BBVA & 18 a 69 & 18 a 69 & 1 & 1,5 \\
\hline Caja Social & 18 a 69 & 18 a 69 & 1,5 \\
\hline
\end{tabular}

Nota: elaboración propia a partir de las páginas web de las entidades bancarias.

Otra de las entidades encargadas de ofrecer crédito hipotecario, es el Fondo Nacional del Ahorro (FNA), creado mediante el Decreto 3118 de 1968, como un ente del Estado, de ámbito financiero, para la administración de las cesantías de los empleados públicos. Posteriormente, fue transformado, mediante la Ley 432 de 1998, en una entidad comercial y financiera bajo la propiedad del Estado, dándole autonomía administrativa y capital independiente. Este proceso tuvo como objetivo principal cubrir las necesidades básicas para la adquisición de vivienda por los hogares. Lo anterior lo convierte 
en otra de las principales alternativas de financiación utilizadas por los hogares. De hecho, el FNA cuenta con un portafolio de servicios que brinda diferentes tipos de opciones y beneficios, siendo el principal requisito ser afiliado. En ese orden, para poder aplicar a un crédito hipotecario, el fondo ofrece dos posibilidades de crédito: a través de ahorro voluntario y a través de cesantías.

El crédito hipotecario a través de ahorro voluntario es un mecanismo utilizado por elFNA, incentivando el ahorro voluntario con destinación a la adquisición de vivienda. Este se constituye mediante contrato, en el cual el futuro solicitante de crédito se compromete con un ahorro voluntario mínimo de doce meses, cumpliendo tres reglas principales: periodicidad en los abonos, monto periódico y monto total. La otra alternativa brindada por el fondo es el crédito hipotecario por cesantías, en el cual se requiere que las cesantías sean trasladadas a la cuenta del FNA, libre de pignoración y embargos de los recursos. En esta modalidad de crédito, el solicitante ahorra la cuota inicial dependiendo del valor del inmueble.

\section{Modelo Logit para el acceso a crédito de vivienda}

\section{Datos}

Con el propósito de evaluar los determinantes de la probabilidad de hacer uso de un crédito hipotecario en Bogotá, se utilizaron los datos de la encuesta multipropósito para esta ciudad (Departamento Administrativo Nacional de Estadística [DANE], 2014). La información utilizó como unidad observacional el hogar, el cual es definido como la persona o el grupo de personas que, independiente de su parentesco, ocupan la totalidad o parte de una unidad de vivienda; además de atender sus necesidades básicas, también comparten un presupuesto en común (DANE, 2007, p. 11). La encuesta contempla 20518 hogares para la ciudad, donde las variables de interés para efectos de esta investigación son el género, la edad, la educación, el ingreso y la vinculación laboral del jefe de hogar.

Para la elaboración del modelo, fue necesario procesar la encuesta, tomando la pregunta que indagaba sobre la fuente de financiamiento utilizada para la compra de vivienda con el objetivo de construir la variable dependiente ${ }^{2}$. Esta variable es de naturaleza dicotómica, tal que los individuos que optaron por la utilización de un crédito hipotecario para financiar su vivienda toman el valor 1 , mientras que las personas que no utilizaron el crédito hipotecario toman el valor o.

$$
y_{i}=\left\{\begin{array}{l}
1 \text { si utilizó crédito hipotecario } \\
0 \text { si no utilizó crédito hipotecario }
\end{array}\right.
$$

Una vez identificada la muestra de la variable dependiente, se filtró la base para identificar a los individuos que respondieron a cada una de las preguntas que fueron tomadas como variables independientes, obteniendo finalmente una muestra de 4611 observaciones. A continuación, se describen las variables que se utilizaron para el modelo:

- Ingreso: representa el valor mensual de los ingresos que tienen los jefes de hogar. Se utilizaron los valores por encima del salario mínimo para 2014, dado los problemas de especificación que se encuentran por debajo de este rango.

- Edad: variable discreta que identifica la edad del jefe de hogar. No se realiza ninguna modificación.

2 Los usuarios de crédito hipotecario contemplados en este estudio son aquellos que se financiaron a través de establecimientos de la banca comercial y el FNA. 
- Género: variable dicotómica que representa el sexo del jefe del hogar. El jefe de hogar mujer es representado con o y el hombre, con 1 . No se tuvieron en cuenta algunas personas que contestaron la condición de intersexual.

- Educación: corresponde al número de años de escolaridad del jefe de hogar. Para la elaboración del modelo se transformó en variable dicotómica, donde 1 representa ser profesional y o no serlo.

- Tipo de contrato laboral: variable cualitativa que representa el tipo de contrato que posee el jefe del hogar (indefinido, fijo, no sabe no responde). Esta variable se transformó en dicotómica para la elaboración del modelo, asignando el valor 1 para contrato a término indefinido, y o para contratos de término fijo y otros tipos de contrato (obra labor, prestación de servicios).

Relaciones básicas entre variables

A continuación, se caracteriza la variable dependiente (hogares que usaron crédito hipotecario), según cada una de las variables independientes. Como se observa en las $\underline{\text { Figuras } 2}$ a $\underline{6}$, existen indicios de que las variables ingreso, educación y tipo de contrato se relacionan con la posibilidad de acceso a crédito hipotecario; en el caso de género no se observa relación; y en el de edad no es evidente.

Figura 2. Porcentaje de hogares que utilizaron o no crédito hipotecario según su nivel de ingreso. Adaptado de DANE (2014).

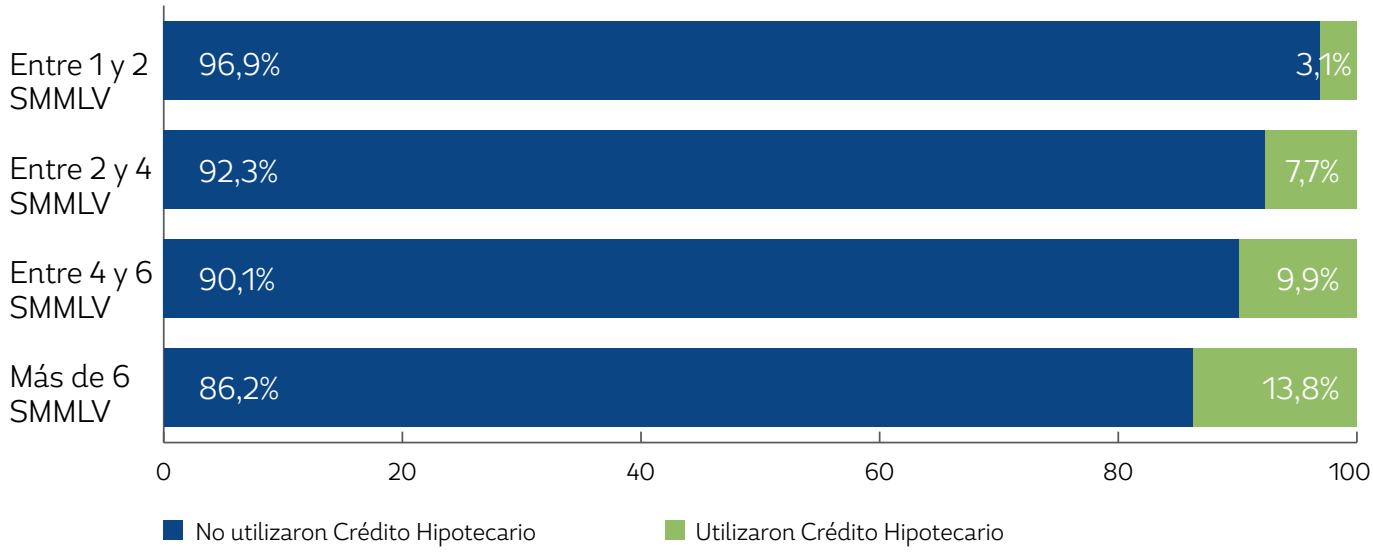

Figura 3. Porcentaje de hogares que utilizaron o no crédito hipotecario según su género. Adaptado de DANE (2014).

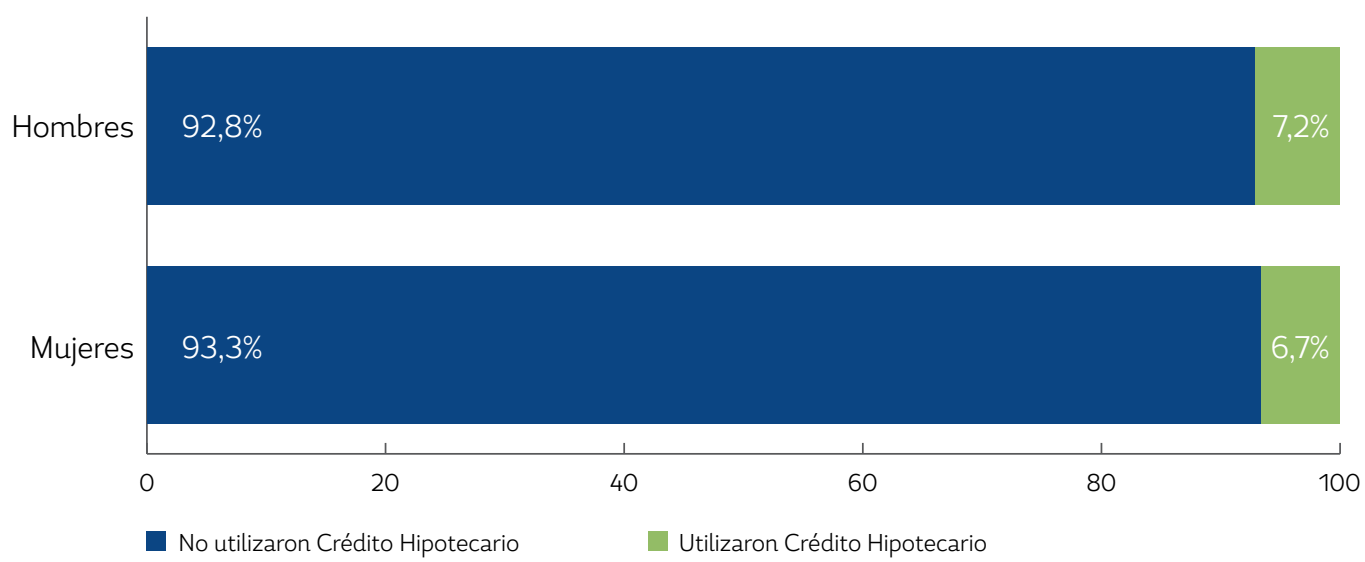


Figura 4. Porcentaje de hogares que utilizaron o no crédito hipotecario según su nivel educativo. Adaptado de DANE (2014).

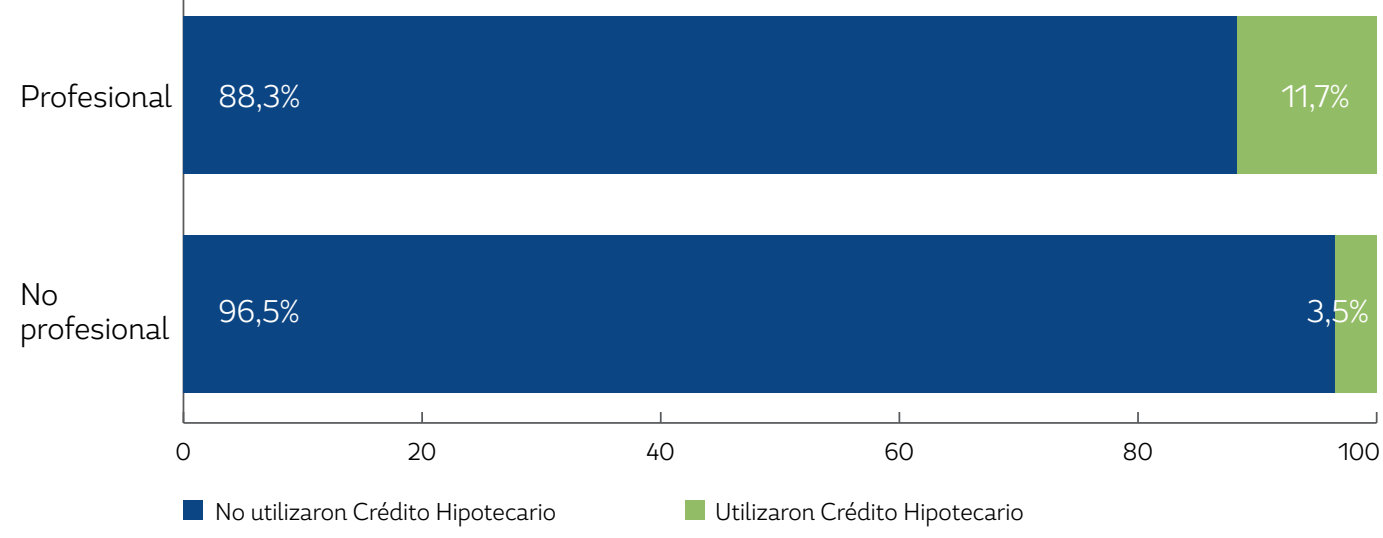

Figura 5. Porcentaje de hogares que utilizaron o no crédito hipotecario según tipo de contrato laboral. Adaptado de DANE (2014).

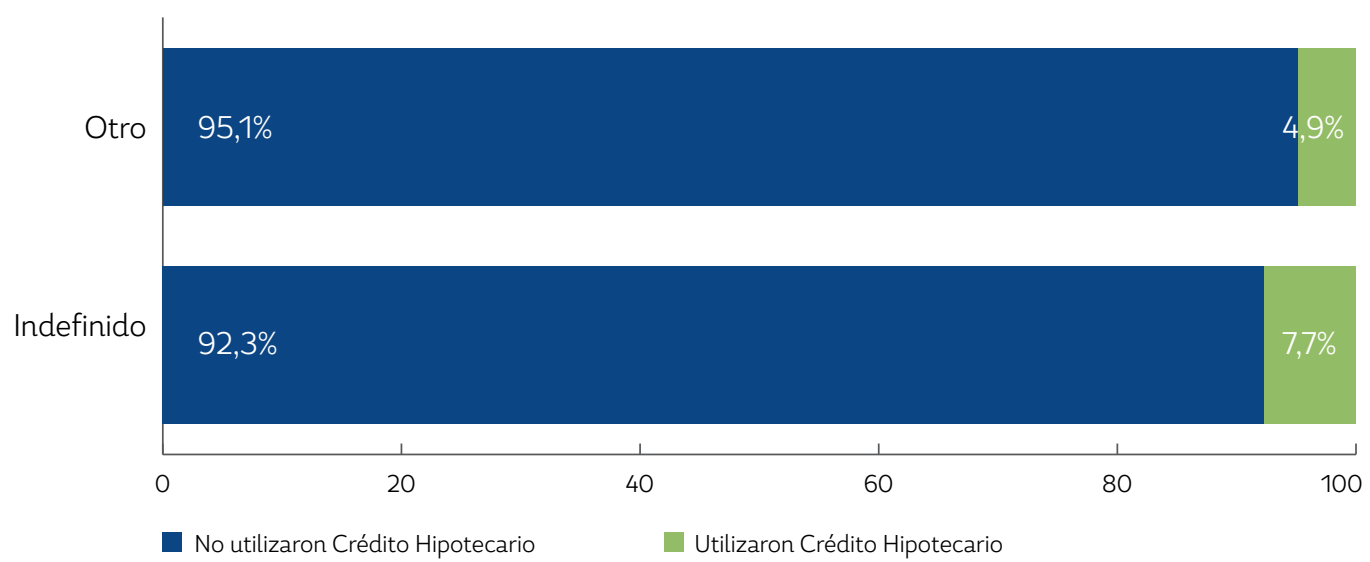

Figura 6. Porcentaje de hogares que utilizaron o no crédito hipotecario según su edad. Adaptado de DANE (2014).

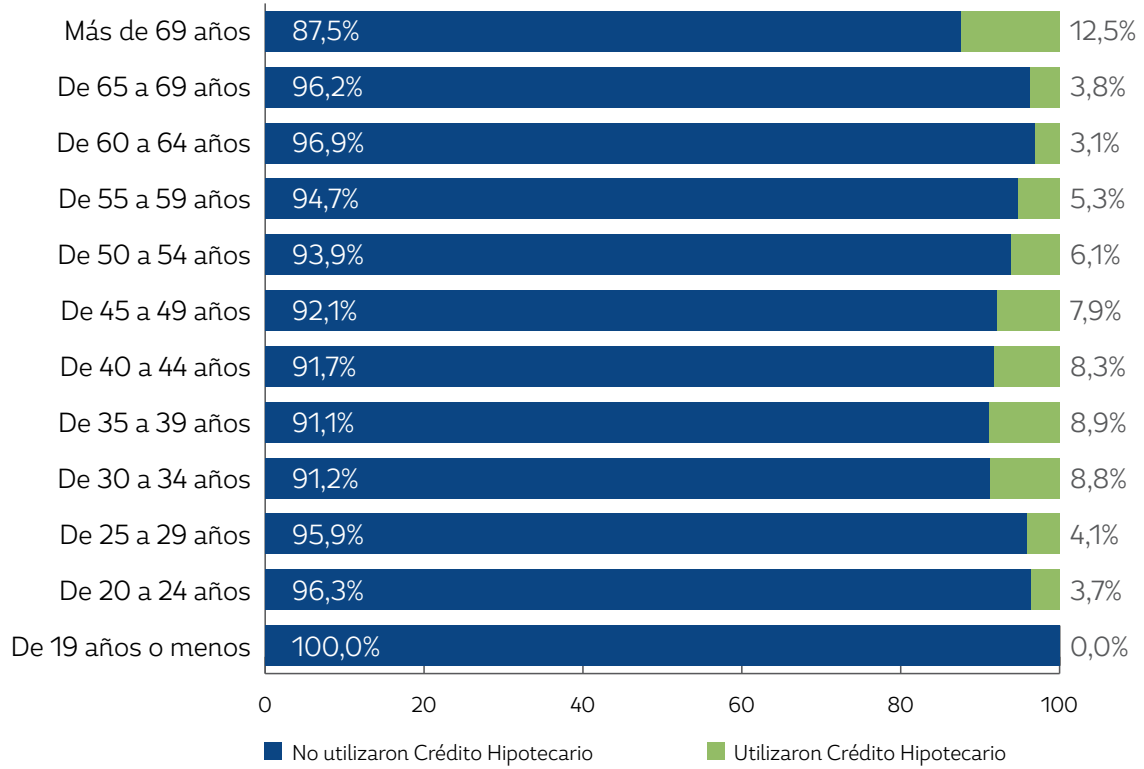


Finalmente, el análisis de correlación entre las variables independientes sugiere poca relación entre ellas, de ahí que no parecen presentar colinealidad (ver $\underline{\text { Anexo 1)}}$ ).

\section{Forma funcional del modelo}

La naturaleza de la variable dependiente lleva a la necesidad de utilizar un modelo de respuesta cualitativa, de ahí que un análisis de regresión lineal tradicional no sea adecuado. En este sentido, los modelos candidatos para la estimación son: Lineal de Probabilidad (MLP), Logit, Probit y Tobit.

Inicialmente, se descartó el modelo Tobit, debido a que se cuenta con información completa sobre las variables dependiente e independientes (Gujarati 83 Porter, 2010, p. 574). En cuanto al MLP, no es adecuado porque, por construcción, no cumple los supuestos básicos del método de mínimos cuadrados ordinarios (MCO) (Gujarati 83 Porter, 2010, p. 552), perdiendo así confiabilidad en las estimaciones. Finalmente, aunque el modelo Probit es una alternativa para analizar problemas donde la variable dependiente es de respuesta cualitativa, en este trabajo se utilizó el modelo Logit por tres razones: 1) la función Logit es matemáticamente fácil de aplicar e interpretar; 2) la mayoría de investigaciones utilizan esta forma funcional en el caso de respuesta binomial; y 3) en general, la bondad de ajuste del modelo es similar al Probit y más adecuado que el MLP (Moscote 8 Rincón, 2012).

En principio, el modelo Logit es no lineal, el cual está representado por la función logística de la forma:

$$
\pi_{i}=\frac{1}{1+e^{-x^{\prime} B}}
$$

Donde denota las variables independientes y $\pi_{i}$ la probabilidad de que la variable dependiente tome al valor de $1\left(E\left(y_{i} / x\right)=1\right)$. No obstante, la función Logit puede transformarse de forma que pueda aplicarse un análisis semejante al de regresión lineal. Despejando $e^{x^{\prime} \beta}$ de la ecuación (2), se obtiene:

$$
\frac{\pi_{i}}{1-\pi_{i}}=e^{-x^{\prime} \beta}
$$

La ecuación (3) es conocida como la transformación Logit de la probabilidad, donde la parte izquierda $\left(\frac{\pi_{i}}{1-\pi_{i}}\right)$ se conoce como el odds ratio o razón de probabilidades (Moscote 8 Rincón, 2012). Obteniendo el logaritmo natural en la ecuación (3), se llega a la ecuación (4), con lo que se establece una relación lineal entre el logaritmo del odds ratio y las variables independientes.

$$
\ln \left(\frac{\pi_{i}}{1-\pi_{i}}\right)=x^{\prime} \beta
$$

Dado que los datos de la encuesta multipropósito se presentan de manera no agrupada (cada observación es un hogar), el método apropiado de estimación de los parámetros es el de máxima verosimilitud, el cual aplica para muestras grandes. Así, el modelo Logit inicial puede expresarse como:

$$
\ln \left(\frac{\pi_{i}}{1-\pi_{i}}\right)=\beta_{0}+\beta_{1} \text { Edad }_{i}+\beta_{2} \text { Genero }_{i}+\beta_{3} \text { Educación }_{i}+\beta_{4} \text { Ingreso }_{i}+\beta_{5} \text { TContrato }_{i}+U_{i}
$$

En la ecuación (5), $\pi_{i}$ indica la probabilidad de que un hogar utilice el crédito hipotecario para el financiamiento de vivienda, por lo tanto, el Logit $\ln \left(\frac{\pi_{i}}{1-\pi_{i}}\right)$ es el logaritmo de la razón de probabilidades entre utilizar el crédito hipotecario o no hacerlo. El modelo permite observar cómo los cambios en las respuestas brindadas por los hogares afectan la probabilidad de adquirir crédito hipotecario; también permite demostrar cuáles de las variables utilizadas son consideradas significativas o no para el modelo (Moscote 8 Rincón, 2012, p. 132). Después de eliminar las variables no significativas, se opta 
por tomar el sistema Stepwise para la elaboración del modelo, el cual plantea realizar interacciones entre las variables significativas para dar resultados más acordes con la investigación.

\section{Bondad de ajuste del modelo Logit con interacciones}

Para los modelos Logit, la prueba R cuadrado como medida de bondad de ajuste no es la más indicada, de ahí que se utilizó otro tipo de pruebas como el porcentaje de aciertos y la Hosmer-Lemeshow (Moscote 8 Rincón, 2012, p. 132).

Para la validación del modelo, existen diferentes pruebas; sin embargo, una de las más indicadas para los modelos Logit con observaciones muy grandes es el porcentaje de aciertos estimados en el modelo, el cual permite determinar la bondad del mismo ${ }^{3}$. Esta prueba consiste en validar la exactitud predictiva a través de comparaciones entre el valor estimado y el observado por grupos; esta no se basa en el valor de la función de verosimilitud, sino en la predicción real de la variable dependiente (Borooah, 2002).

Tabla 3. Porcentaje de aciertos estimados en el modelo

\begin{tabular}{l|c|c|c}
\multicolumn{1}{c|}{ Índice } & Valor & Índice & Valor \\
\hline Tasa de aciertos & $92,97 \%$ & Sensibilidad & $0,00 \%$ \\
\hline Tasa de errores & $7,03 \%$ & Tasa de falsos 0 & $7,03 \%$ \\
\hline Especificidad & $100,00 \%$ & Tasa de falsos 1 & $100 \%$ \\
\hline
\end{tabular}

Fuente: elaboración propia a partir de los datos obtenidos del modelo econométrico.

El modelo Logit tiene un buen porcentaje de aceptabilidad para el total de las predicciones, la cual se refleja en las altas tasas de acierto en la totalidad del modelo.

De otro lado, se utilizó la prueba de Hosmer-Lemeshow, la cual pretende comprobar si el modelo propuesto puede explicar las observaciones. Su elaboración consiste en dividir las observaciones en $j$ grupos o intervalos (generalmente 10 observaciones), del mismo tamaño, dividiendo la probabilidad de ocurrencia de la hipótesis planteada; es decir, se valida intervalo por intervalo (esperado y observado) para todos los resultados de la variable dependiente (Borooah, 2002). En contraste, el valor de probabilidad para el modelo estimado es de 0,0623, lo que indica que presenta un buen ajuste, dados los datos brindados por la encuesta multipropósito.

\section{Resultados}

Los resultados arrojaron la siguiente ecuación estimada:

$$
\ln \left(\frac{\pi_{i}}{1-\pi_{i}}\right)=-3.3926-0.0103 \text { Edad }+1.1156 \text { Educacion }+5,40^{E-08} \text { Ingreso }+0,4248 \text { TContrato }+U
$$

En la primera estimación del modelo, la variable género no resultó significativa al $5 \%$ (ver Anexo 2), de ahí que se excluya en la ecuación (6). Por su parte, las demás variables consideradas parecen tener la influencia esperada sobre el uso de crédito hipotecario: cuanto mayor edad del jefe de hogar menor propensión a utilizar crédito hipotecario; un mayor ingreso implica mayor probabilidad de utilizar el crédito; y el hecho de contar con contrato a término indefinido y un nivel de educación superior incrementa las probabilidades de utilizar el crédito para adquirir vivienda.

3 El valor de la prueba "c" generalmente es 0,5. Con esto, se determina que, si el valor de la predicción es igual a 1 o 0, es de 0,5, puesto que parece lógico que la predicción sea 1 cuando el modelo dice que es más probable obtener un 1 que un 0. 
Ante la posibilidad de encontrar otro tipo de relaciones no lineales entre las variables independientes y la dependiente, se realizó el proceso Stepwise, el cual permitió identificar interacciones entre las variables regresadas. De esta manera, se estimó la siguiente ecuación:

$$
\begin{aligned}
& \ln \left(\frac{\pi_{i}}{1-\pi_{i}}\right)=-4.0387-0.0159 \text { Edad }+2.1319 \text { Educación }-0.0173 \text { TContrato }+ \\
& 5.85^{E-08} \text { Ingreso }-0.0391 \text { Educación } * \text { Edad }+0.6993 \text { Educación } * \text { TContrato }+U_{1}
\end{aligned}
$$

En la ecuación se puede evidenciar que existen variables con relación positiva, las cuales, al aumentar, otorgaron mayor posibilidad de acceder a crédito; este es el caso de la edad, la educación y el gasto. Ahora, el tipo de contrato no es significativa individualmente, pero sí lo es mediante interacción con la educación; en este sentido, el tipo de contrato indefinido mejora las probabilidades de utilización del crédito de vivienda, siempre y cuando esto esté acompañado de mayor nivel de educación. No obstante, la interacción entre educación y edad también es significativa, cuyo coeficiente negativo sugiere que los jefes de hogar con mayor edad, y que al mismo tiempo presentan mayor nivel educativo, tienden a hacer menos uso del crédito hipotecario.

El modelo estimado presenta una aceptable bondad de ajuste y permite realizar predicciones respecto a la probabilidad de hacer uso del crédito hipotecario por parte de determinado jefe de hogar.

Interpretación a partir de escenarios

Con base en la ecuación (7), la Tabla 4 presenta la estimación de probabilidad de hacer uso de crédito hipotecario ante diferentes escenarios, sobre las características del jefe de hogar.

Tabla 4. Escenarios de probabilidad de utilización de un crédito hipotecario

\begin{tabular}{c|c|c|c|c|c}
\hline Escenario & Edad & Educación & Tipo decontrato & Ingreso & Probabilidad \\
\hline 1 & 30 & 0 & 0 & 1000000 & $1,15 \%$ \\
\hline 2 & 40 & 0 & 0 & 1000000 & $0,98 \%$ \\
\hline 3 & 30 & 1 & 0 & 1000000 & $2,94 \%$ \\
\hline 4 & 30 & 0 & 1 & 1000000 & $1,13 \%$ \\
\hline 5 & 30 & 0 & 0 & 2000000 & $1,21 \%$ \\
\hline 6 & 40 & 1 & 0 & 1000000 & $1,72 \%$ \\
\hline 7 & 30 & 1 & 1 & 1000000 & $5,64 \%$ \\
\hline
\end{tabular}

Fuente: elaboración propia a partir de los datos obtenidos del modelo econométrico.

Es posible identificar, mediante tales escenarios, el efecto concreto de cada una de las variables consideradas sobre el uso de crédito hipotecario que hacen los hogares. En particular, partiendo de un caso referencia (escenario 1) donde el jefe de hogar tiene 30 años de edad, no profesional, sin contrato laboral a término indefinido y un ingreso de \$1 ooo ooo mensuales, tiene una probabilidad de uso del crédito hipotecario de 1,15\%. Los escenarios 2, 3, 4 y 5 modifican las variables edad, educación, tipo de contrato e ingreso, evidenciando que la variable educación es aquella que presenta mayor importancia en el modelo. En particular, si el individuo referencia pasa a tener educación profesional, su probabilidad de uso de crédito hipotecario aumenta a 2,94\%, pero si, además, obtiene un contrato a término indefinido, su probabilidad será cercana a 5,64\%. 
Se observa que la edad, el tipo de contrato y el ingreso, de manera individual, no son variables muy determinantes en el uso del crédito hipotecario; sin embargo, tienden a ser factores complementarios. En otras palabras, la edad del jefe de hogar es una variable que afecta el uso del crédito de manera inversa, lo cual es consistente con la idea del ciclo vital en el consumo (Modigliani, 1986); no obstante, tal efecto es mayor en las personas con educación profesional. Por su parte, el tipo de contrato a término indefinido puede favorecer el uso de crédito de vivienda, siempre que el jefe de hogar cuente con educación profesional, pero lo afectaría de manera inversa (aunque leve) en hogares sin educación. Este último hallazgo puede explicarse, en parte, por los factores enunciados por Gonzales (2005), según los cuales la insuficiente capacidad adquisitiva de los hogares limita su demanda efectiva de vivienda.

\section{Conclusiones}

El alto costo de la vivienda y las condiciones socioeconómicas de muchos hogares colombianos han hecho del crédito hipotecario una herramienta esencial que apalanca el acceso a la vivienda; sin embargo, este análisis sugiere que las actuales políticas no suelen ser efectivas en el mercado de vivienda, cuya prioridad pasa por promover la demanda sin atacar estructuralmente las limitantes de ingreso, educación y empleo de la población. En efecto, el marco normativo del financiamiento de vivienda en el país se ha concentrado en mecanismos de financiación, pero sin promover alternativas eficaces que hagan asequible la vivienda para muchas familias.

Aunque existe una amplia gama de opciones para que un hogar elija un esquema de financiación bancaria, tanto en UVR como en pesos, todas ellas requieren unas garantías mínimas de mediano y largo plazo, tanto de nivel de ingresos como de estabilidad laboral. Para aquellos hogares que no cuentan con un ingreso suficiente, las restricciones impuestas por el mercado financiero siguen siendo muy fuertes, y esto es evidente ante la necesidad de contar con un ahorro que alcance al menos el $30 \%$ del valor del inmueble para hacer uso del crédito hipotecario. De otra parte, en cuanto a la estabilidad laboral, es una condición normativa para otorgar créditos hipotecarios por parte de las entidades bancarias, siendo un elemento que pocas veces es ofrecido por el mercado.

Algunas variables analizadas empíricamente en esta investigación, tales como la edad, el empleo y los ingresos, son tenidas en cuenta en los requisitos que imponen las entidades bancarias en Colombia; No obstante, otras no lo son de manera directa, es el caso de aspectos culturales o idiosincráticos. Como una variable proxy de estos elementos, puede sugerirse el nivel de educación, que, como se observó en el ejercicio empírico, es uno de los principales determinantes que llevan a un hogar a hacer uso efectivo del crédito hipotecario para su acceso a vivienda. En este sentido, mediante la estimación del modelo Logit, se valida la importancia del ingreso, la edad, la educación y el empleo en el uso del crédito hipotecario, pero tal importancia se potencializa cuando el jefe de hogar cuenta con un nivel educativo profesional.

Concretamente, en el caso de la edad, son los jóvenes con educación profesional los que presentan mayor probabilidad de uso de crédito, y en el caso del empleo a término indefinido, este es importante en la medida que el jefe de hogar cuente con educación profesional. Así, un hallazgo central es que la educación se ha convertido en un aspecto fundamental, pues no se trata solamente de acceder a un crédito hipotecario, sino de tener las condiciones efectivas para soportar dicho crédito durante un plazo de hasta 30 años. 
Desde un enfoque teórico, se evidencia que las asimetrías de información propias del mercado, al no reconocer suficientemente el grado de solvencia y cumplimiento de un hogar, conllevan la incapacidad del sistema financiero de dar oportunidades de acceso, de manera equitativa, a toda la población. De otro lado, la teoría del ciclo vital parece tener relevancia en la medida que los hogares cuyo jefe de hogar tiene mayor edad no suelen hacer uso del crédito hipotecario para obtención de vivienda propia. Se puede entender que muchas personas mayores ya cuentan con vivienda propia obtenida a través de otras alternativas, y en sus prioridades no se encuentra el endeudamiento para obtención de vivienda; aun así, hace parte de una agenda futura el identificar si estos patrones se dan por restricciones del mercado o por elección de los hogares.

En términos prácticos, este estudio es de alta relevancia, tanto para las entidades que ofrecen alternativas de financiamiento de vivienda como para los hacedores de política pública. Es fundamental, para las entidades financieras, considerar factores más allá de lo estrictamente económico, por lo que se deben establecer criterios de identificación de variables como la cultura de pago del individuo y los incentivos. No obstante, es evidente que sigue existiendo una dicotomía entre las garantías requeridas por las entidades y el derecho de vivienda digna que consagra la ley. En este último aspecto, los hacedores de política son los encargados de fijar mecanismos de solidaridad y subsidiariedad que no se limiten a la mera regulación, sino que propendan por la formalidad laboral y la educación, especialmente de la población joven que recién se incorpora en la dinámica del mercado de vivienda. 


\section{Referencias}

Akerlof, G. A. (1970). The market for "Lemons": Quality uncertainty and the market mechanism. Quarterly Journal of Economics, 84(3), 488-500.

Ando, A., \& Modigliani, F. (1963). The life cycle hypothesis of saving: Aggregate implications and Tests. The American Economic Review, 4(53), 55-84.

Asamblea Nacional Constituyente. (1991). Constitución Política de Colombia (2. ㄹ ed.). Bogotá: Legis.

Baena, L. G. (2017). Fiducia inmobiliaria. Tensión entre la autonomía privada, el derecho a la vivienda digna y el derecho del consumo. Bogotá: Universidad Externado de Colombia.

Borooah, V. K. (2002). Logit and probit. Ordered and Multinomial models. Thousand Oaks, CA: Sage Publications.

Camargo, A., \& Hurtado, A. (2011). Vivienda y pobreza: una relación compleja. Cuadernos de Vivienda y Urbanismo, 4(8), 224-246.

Camelo, M., \& Ocampo, I. (2016). Análisis de vivienda en Bogotá: Un enfoque desde la oferta y la demanda. Revista Finanzas y Política Económica, 8(1), 105-122.

Carvajal, P., García, I., \& Cotte, A. (2015). Determinantes socio-económicos y financieros del acceso a vivienda de interés prioritario: un estudio para el caso colombiano durante el período 2009-2012. Revista investigación operacional, 36(2), 169-177.

Clavijo, S., Muñoz, S., \& lanna, M. (2004). La vivienda en Colombia: sus determinantes socio-económicos y financieros. Borradores de Economía, 300. Recuperado de http://www.banrep.gov.co/sites/default/ files/publicaciones/pdfs/borra300.pdf

Connolly, P. (1997). El financiamiento de vivienda en México [en línea]. Recuperado de http://infonavit.janium. net/janium /UAM/ Documentos/19752.pdf

Daphnis, F. (2004). Housing microfinance: towards a definition. En F. Daphnis y B. Ferguson, (eds.), Housing Microfinance: a guide to practice (pp. 1-1). Bloomfield, CT : Kumarian Press, Inc.

De Leeuw, F. (1971). The demand for housing: a review of crosssection evidence. The Review of Economic and Statistics, 53(1), 1-10.

Departamento Administrativo Nacional de Estadística. (2007). La información estadística del Censo General 2005 y su pertinencia en la planeación del desarrollo local y regional. Cartilla de conceptos básicos e indicadores demográficos. Recuperado de https://www.dane.gov.co/files/etnicos/cartilla_quibdo.doc

Departamento Administrativo Nacional de Estadística. (2014). Encuesta multipropósito. Recuperado de https://www.dane.gov.co/index.php/estadisticas-por-tema/pobreza-y-condiciones-de-vida/encuesta-multiproposito/encuesta-multiproposito-2014

Díaz, G. (2011). Las imperfecciones del mercado de créditos, la restricción crediticia y los créditos alternativos. Revista CIFE, 17(12), 103-133.

Durán, A. I. (2013). Propuesta de acceso a financiamiento para la adquisición de vivienda a familias con actividad laboral de temporero agrícola en la región del Maule. Santiago: Universidad de Chile.

Fallis, G., \& Smith, L. B. (1984). Uncontrolled prices in a controlled market: the case of rent controls. The American Economic Review, 4(1), 193-200.

Ferguson, B., \& Navarrete, I. (2003). New approaches to progressive housing in Latin America: A key to habitat programs and policy. Habitat International, 27, 309-323.

Ferguson, B., \& Smets, P. (2010). Finance for incremental housing; current status and prospects for expansion. Habitat International, 34, 288-298.

Friedman, M. (1957). A theory of the consumption function. Princeton, NJ: Princeton University Press.

Fruet, I. V., \& Muñoz, G. A. (2015). Paraguay: una propuesta de financiamiento de viviendas para los segmentos de ingresos medios-bajos. Revista de La CEPAL, 117, 87-107. 
Gonzales, G. (2005). El crédito hipotecario y el acceso a la vivienda para los hogares de menores ingresos en América Latina. Revista de la Cepal, 86, 115-128.

Gujarati, D., \& Porter, D. (2010). Econometría (5. a ed.). México D. F.: McGraw-Hill.

Gurley, I., \& Shaw, E. (1955). Financial Aspects of Economic Development. American Economic Review, 45, 515-538.

Hoff, K., \& Stiglitz, J. (1981). Introduction: Imperfect information and rural credit markets - puzzles and policy perspectives. The World Bank Economic Review, 4(3), 235-250.

Hood, I. (1999). The Determinants of Home Ownership: An Application of Human Capital Investment Theory to the Home Ownership Decision. Honors Projects. Recuperado de http://digitalcommons.iwu.edu/ econ_honproj/71

Modigliani, F. (1986). The life cycle hypothesis of saving, the demand for wealth and the supply of capital. Social Research, 33(2), 160-217.

Moscote, O., \& Rincón, W. (2012). Modelo Logit y Probit: un caso de aplicación. Comunicaciones de Estadística, 5(2), 123-133.

Murcia, A. (2007). Determinantes del acceso al crédito de los hogares colombianos. Borradores de Economía, 449.

Poterba, J. M. (1984). Tax subsidies to owner-occupied housing: an asset-market approach. The Quarterly Journal of Economics, 99(4), 729-752.

Rodriguez, A., \& Sugranyes, A. (2012). Vivienda privada de ciudad. Revista de Ingeniería, 35, 100-107.

Rojas, M., Bran, N., \& Rincón, C. (2013). Modelos de financiación de vivienda, casos México, Chile y Colombia. Revista Ingenierías Universidad de Medellín, 12(22), 97-108.

Serageldin, M., \& Driscoll, J. (2000). Housing microfinance initiatives: synthesis and regional summary: Asia, Latin America and SubSaharan Africa with selected case studies. Cambridge, MA: Center for Urban Development Studies/Harvard University Graduate School of Design.

Serrano, R. I. (2012). Financiamiento de vivienda. Revista de Ingeniería, 35, 61-78.

Stiglitz, J., \& Weiss, A. (1981). Credit rationing in markets with imperfect information. The American Economic Review, 71(3), 393-410.

Superintendencia Financiera de Colombia. (13 de septiembre de 2000a). Circular Externa 068 de 2000: Procedimiento de Liquidación de Créditos Hipotecarios de Vivienda. Recuperado de https://www.superfinanciera.gov.co/descargas?com=institucional\&name=pubFile83447\&downloadname=ce068.rtf

Superintendencia Financiera de Colombia. (29 de diciembre de 2000b). Circular Externa 085 de 2000: Disposiciones aplicables a los créditos de vivienda. Recuperado de https://www.superfinanciera.gov.co/ descargas?com=institucional\&name=pubFile83357\&downloadname=ce085.rtf

Torres, C., Rincón, I., \& Vargas, I. (2009). Pobreza urbana y mejoramiento integral de barrios en Bogotá. Bogotá: Universidad Nacional de Colombia.

Vickrey, W. (1961). Counterspeculation, Auctions and Competitive Sealed Tenders. Journal of Finance, 16, 8-37.

Villareal, R. (2010). El mercado de crédito colombiano, un análisis de las reformas financieras. Equidad y Desarrollo, 14, 67-83.

Young, C. (2007). Housing microfinance: designing a product for the rural poor. Institute for Financial Management and Research, Centre for Micro Finance, Working Paper Series, 19. 


\section{Anexo 1}

Matriz de correlación entre variables independientes

\begin{tabular}{l|c|c|c|c|c} 
& Edad & Educación & Género & Ingreso & Tcontrato \\
\hline Edad & 1,00000 & 0,05804 & 0,01676 & 0,10741 & 0,05653 \\
\hline Educación & 0,05804 & 1,00000 & $-0,10235$ & 0,47972 & 0,02252 \\
\hline Género & 0,01676 & $-0,10235$ & 1,00000 & 0,05959 & 0,04161 \\
\hline Inóreso & 0,10741 & 0,47972 & 0,05959 & 1,00000 & 0,06201 \\
\hline Tcontrato & 0,05653 & 0,02252 & 0,04161 & 0,06201 & 1,00000 \\
\hline
\end{tabular}

Nota: Adaptado de DANE (2014)

\section{Anexo 2}

\section{Estimación de modelos}

Resultados de la estimación Logit para el modelo inicial

\begin{tabular}{l|c|c|c|c}
\multicolumn{1}{c|}{ Variable } & Coeficiente & Error Std & Z estadística & Probabilidad \\
\hline Edad* & $-0,010323$ & 0,005532 & $-1,865914$ & 0,0621 \\
\hline Género & 0,130051 & 0,129198 & 1,006606 & 0,3141 \\
\hline Educación*** & 1,115556 & 0,138566 & 8,050723 & 0,0000 \\
\hline Ingreso*** & $5,40 E-08$ & $1,42 E-08$ & 3,793934 & 0,0001 \\
\hline TContrato** & 0,424816 & 0,158004 & 2,688643 & 0,0072 \\
\hline C*** & $-3,392585$ & 0,284487 & $-1,192525$ & 0,0000 \\
\hline
\end{tabular}

Nota: Adaptado de DANE (2014). *Nivel de significancia al 0,1. **Nivel de significancia al 0,05. ***Nivel de significancia al 0,01.

Resultados de la estimación Logit con interacciones (STEPWISE)

\begin{tabular}{l|c|c|c|c}
\multicolumn{1}{c|}{ Variable } & Coeficiente & Error Std & Z estadística & Probabilidad \\
\hline Edad* & 0,015985 & 0,009587 & 1,667279 & 0,0955 \\
\hline Educación*** & 2,131948 & 0,559558 & 3,810060 & 0,0001 \\
\hline Ingreso*** & $5,85 E-08$ & $1,42 E-08$ & 4,134269 & 0,0000 \\
\hline TContrato & $-0,017276$ & 0.249060 & $-0,069364$ & 0,9447 \\
\hline Educación x Edad*** & $-0,039124$ & 0,011808 & $-3,313387$ & 0,0009 \\
\hline Educación x TContrato** & 0,699256 & 0,322812 & 2,166138 & 0,0303 \\
\hline C*** & $-4,038665$ & 0,454502 & $-8,885905$ & 0,0000 \\
\hline
\end{tabular}

Nota: Adaptado de DANE (2014). *Nivel de significancia al 0,1. **Nivel de significancia al 0,05. ***Nivel de significancia al 0,01. 